Acta Crystallographica Section D

Biological

Crystallography

ISSN 0907-4449

Ted Baker ${ }^{\mathrm{a}}$ and

Zbigniew Dauter ${ }^{b}$

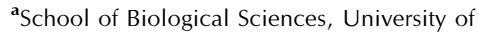
Auckland, Private Bag 92-019, Auckland, New Zealand, and ${ }^{\mathbf{b}}$ National Cancer Institute, Brookhaven National Laboratory, Biology Department, Bldg 463, Upton, NY 11973, USA

\section{Acta D ten years on}

This year marks the tenth anniversary of the establishment of Section D of Acta Crystallographica. To recognize this milestone we will be featuring, over the next few issues, invited articles from a number of authors who have made significant contributions to biological crystallography, and to Acta Crystallographica. Fittingly, the four articles in the present issue highlight two different aspects of the extraordinary power of crystallography. Two papers, from Michael Rossmann and from Jack Johnson, show the spectacular way in which crystallography can address the structures of large biological assemblies. This was foreshadowed more than 50 years ago, when the first viruses were crystallized, but required that suitable methods be worked out for handling such large structures and exploiting the symmetry within them. Two other papers, from Peter Lindley and John Helliwell, focus on the chemistry of biological molecules, in one case examining fine atomic detail, in the other providing the chemical and structural basis for a

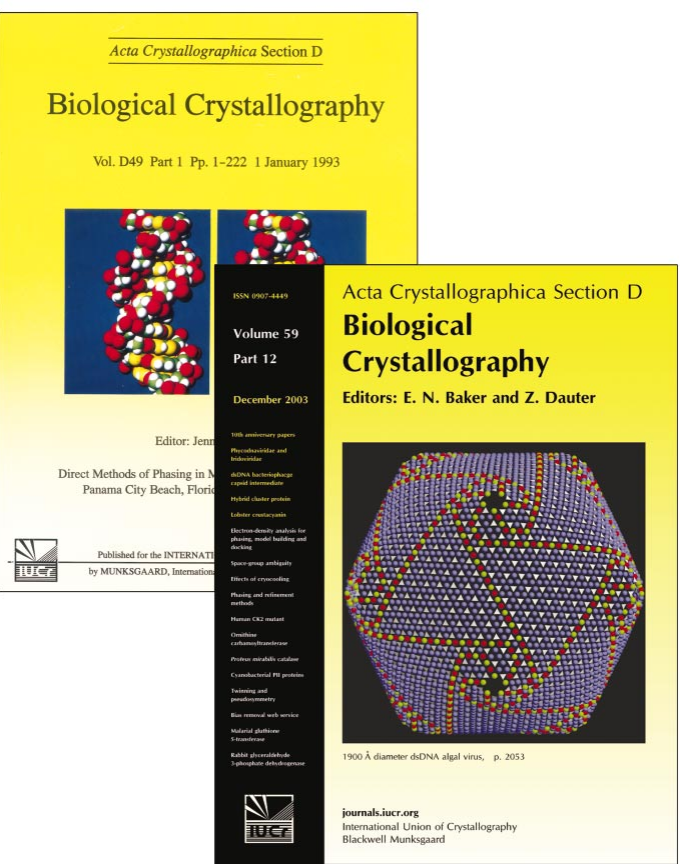

Acta D in 1993 and 2003. remarkable colour change in biology. In each case, the role of crystallography is to give chemical or biological insights.

What changes have we seen over the past ten years? First, there has been a huge upsurge in the development of faster and more powerful methods. In parallel, macromolecular structure has become integral to many areas of biological research and of great importance to biotechnology and medicine. This trend is set to continue. Every issue of Acta $D$ brings new approaches to phasing, model building, refinement or crystallization, and the repertoire of journals that publish crystallographic results is increasing all the time.

How will this affect Acta Crystallographica over the next ten years? The increasing numbers of structural biologists and the dramatic advances in the speed and ease of macromolecular structure determination will bring a huge expansion in the quantity of structural data for publication. Some will come from structural genomics, others from drug development or functional investigations. Some will be ground-breaking, but others will be 'routine' analyses, paralleling the changes that took place in chemical crystallography some 20-30 years ago. 
These will bring pressures on journals, with increasing demand for space.

Acta Crystallographica is a 'society' journal, published by the International Union of Crystallography. As such, it carries moral force in the field, as evidenced for example by its leading role in advocating and ensuring appropriate deposition policies, on behalf of the worldwide community. We do not have the resources of large publishing houses, and must walk a fine line between financing new initiatives and maintaining high quality but affordable journals for the benefit of all crystallographers. No journal can stand still, however, and in the coming months we hope to announce a new initiative that will increase our commitment to biological crystallography, and which we hope will have the enthusiastic support of all our readers. 